\title{
Root resorption during orthodontic treatment
}

\author{
Abstracted from \\ Weltman B, Vig KW, Fields HW, Shanker S, Kaizar EE. \\ Root resorption associated with orthodontic tooth movement: a systematic review. \\ Am J Orthod Dentofacial Orthop 2010; 137: 462-476. \\ Address for correspondence: Katherine WL Vig, 4088 F Postle Hall, \\ Ohio State University College of Dentistry, 305 W 12th Ave, PO Box 182357 \\ Columbus, OH 43218-2357, USA. E-mail: vig.2@osu.edu <mailto:vig.2@osu.edu>
}

\section{Questions: What is the effect of orthodontic treatment on root resorption?}

Data sources Medline, Embase, LILACS, The Cochrane Library (Cochrane Database of Systematic Reviews, CENTRAL, and Cochrane Oral Health Group Trials Register) Web of Science, EBM Reviews, Computer Retrieval of Information on Scientific Project (CRISP, www. crisp.cit.nih.gov), On-Line Computer Library Center (www.oclc. org), Google Index to Scientific and Technical Proceedings, PAHO (www.paho.org), WHOLis (www.who.int/library/databases/en), BBO (Brazilian Bibliography of Dentistry), CEPS (Chinese Electronic Periodical Services), Conference materials (www.bl.uk/services/bsds/ dsc/conference.html), ProQuest Dissertation Abstracts and Thesis database, TrialCentral (www.trialscentral.org), National Research Register (www.controlled-trials.com), www.Clinicaltrials.gov and SIGLE (System for Information on Grey Literature in Europe).

Study selection Randomised controlled trials including split mouth design, recording the presence or absence of external apical root resorption (EARR) by treatment group at the end of the treatment period. Data extraction and synthesis Data were extracted independently by two reviewers using specially designed and piloted forms. Quality was also assessed independently by the same reviewers

Results After evaluating titles and abstracts, 144 full articles were obtained of which 13 articles, describing 11 trials, fulfilled the criteria for inclusion. Differences in the methodological approaches and reporting results made quantitative statistical comparisons impossible. Evidence suggests that comprehensive orthodontic treatment causes increased incidence and severity of root resorption, and heavy forces might be particularly harmful. Orthodontically induced inflammatory root resorption is unaffected by archwire sequencing, bracket prescription, and self-ligation. Previous trauma and tooth morphology are unlikely causative factors. There is some evidence that a two- to three-month pause in treatment decreases total root resorption. Conclusions The results were inconclusive in the clinical management of root resorption, but there is evidence to support the use of light forces, especially with incisor intrusion.

\section{Commentary}

External apical root resorption (EARR) can be a significant sequela of orthodontic treatment and in the most severe cases may threaten the longevity of the teeth. Identification of the factors contributing to EARR during orthodontic treatment is therefore essential in order to minimise the incidence and severity of root resorption. This systematic review therefore addresses a very important question: what are the effects of orthodontic treatment factors on root resorption?

This is a well-designed systematic review, using methods based on established Cochrane recommendations for systematic reviews. The objectives of the review are clearly stated. A rigorous and comprehensive search strategy was formulated to identify all potentially relevant studies, including unpublished and foreign-language trials. Eleven studies were identified as suitable for inclusion in the review. All were randomised controlled trials, but the methodological quality of these varied. Some were split mouth studies with follow-up times as short as nine weeks. The validity of these studies in relation to comprehensive orthodontic treatment is questionable and their inclusion in the review could lead to potentially misleading results. The authors do, however, draw attention to the limitations of these trials in their discussion and point out that no firm conclusions can be drawn. Some of the studies included in the review, although of high quality, were designed to investigate the effect of different fixed appliance systems or archwire sequences, and thus did not have a control group. The protocol for the systematic review states that trials included should have a control group who were not subjected to active orthodontic force. These trials should, perhaps, have been excluded from the review, as, strictly speaking, they did not meet the inclusion criteria.

In spite of the potential minor shortcomings, this is a good quality systematic review using thorough methodology. The potential problems with the evidence found are discussed at length by the authors and the conclusions they make are based on sound evidence. The key point to take from this review is that, whilst orthodontic treatment is associated with an increased incidence and severity of EARR, it is not currently possible to determine which patients are at particular risk. Until better evidence becomes available, all prospective orthodontic patients should be warned of the possibility of root resorption as part of the consent process. Additionally, pretreatment radiographs should be taken for all patients, to act as a baseline against which any future resorption can be measured.

Sally Walker

Cumberland Royal Infirmary, Carlisle, UK

Evidence-Based Dentistry (2010) 11, 88. doi:10.1038/sj.ebd.6400743 